\title{
Review of: "Prefrontal tDCS attenuates counterfactual thinking in female individuals prone to self-critical rumination"
}

\author{
Jacopo Lisoni ${ }^{1}$, Antonio Vita ${ }^{2}$ \\ 1 ASST Spedali Civili \\ 2 University of Brescia
}

Potential competing interests: The author(s) declared that no potential competing interests exist.

The goals of the present Review are (I) to discuss some preliminary definitions about rumination and Counter-Factual Thoughts according to current literature; (II) to discuss the results presented by Alleart and colleagues and other tDCS studies concerning rumination; and (III) to discuss the implications of these findings regarding other psychiatric conditions, such as Borderline personality Disorder.

First, to best understand the relevance of results provided by Alleart et al, we briefly summarize the rumination concept and its clinical importance in psychiatric disorders. Among different emotion-regulation strategies, rumination refers to a repetitive, recurrent, and uncontrollable negative style of thinking ${ }^{[1]}$ about selfness, feelings, personal concerns, and upsetting experiences ${ }^{[2]}$ i.e, a distorted cognitive process described as adysfunctional coping strategy strictly linked to emotion regulation and metacognition [3]. Ruminative thoughts critically occur in a plethora of psychiatric disorders such as mood, anxiety, personality, eating, and substance use disorders ${ }^{[4]}$ : in this scenario, maladaptive ruminations could be interpreted as a transdiagnostic pathological mediator of mental health vulnerability and outcome of psychopathology [3] [5]. More precisely, rumination can exacerbate psychopathology, magnifying current negative mood states (such as sadness and anger) through the amplification of vicious repetitive cycles between negative mood and negative cognition ${ }^{[6]}$, then interfering with successful problem-solving and leading to a reduced sensitivity to change ${ }^{[7]}$. Neurobiologically, rumination seems to be related to deficiencies in top-down executive control process in term of a decreased activation of the prefrontal cortex, particularly of the dorsolateral prefrontal cortex (DLPFC) ${ }^{[8]}[9]$. Moreover, at cognitive level, the relationship between recurrent negative thinking and altered cognitive control ${ }^{[10]}$ seems to be mediated by distortion of different processes such as working memory [11] and attention [12].

Counter-Factual Thoughts (CFT) are mental representations of alternatives to past events, actions, or states ${ }^{[13]}$. Deficiencies in CFT are related to difficulties in behavioral and affective modulation, disturbing the normal decision-making process. This style of thinking is more evident especially in patients suffering from psychiatric disorder, in which rumination rates are higher. Although rumination and CFT are conceptually discrete, some Authors observed that dysfunctional CFT could be the fodder for rumination, 
thus leading to greater levels of depressive and anxious states [13].

In this context, since Non-Invasive Brain Stimulation (NIBS) was found to improve cognition both in healthy subjects ${ }^{[14]}$ and in psychiatric disorders ${ }^{[15]}$, Alleart and colleagues highlighted the potential effects of tDCS intervention improving rumination and CFT by enhancing DLPFC activity in healthy volunteers ${ }^{[16]}$. Based on the premise that rumination-prone individuals are more frequently experiencing CFT and regret, the goals of the present study were (I) to investigate whether anodal tDCS over the left DLPFC momentarily attenuates CFT and regret, and (II) to explore psychophysiological correlates (skin conductance and heart rate) of tDCS effect in response to varying choice outcomes related to goal progress. The study involved eighty female healthy individuals and consisted of one 20-min tDCS session $(2 \mathrm{~mA})$ in which direct current was delivered targeting the left DLPFC (F3) with anodal stimulation coupled with cathodal right OFC (Fp2) stimulation. An adaptation of the Devil's task, originally designed to assess risk preferences through choices made in a switch-pulling game, was performed to evaluate tDCS influence on decision-making process. Authors observed that, among rumination-prone individuals, anodal (versus sham) tDCS was associated with decreased CFT and attenuated psychophysiological reactivity to differential choice outcomes. Conversely, among low rumination-prone individuals, anodal (versus sham) tDCS was associated with both increased CFT and regret, but in absence of any effects on psychophysiological reactivity. The Authors argued that habitual inclination towards self-critical rumination was a leading factor accounting for DLPFC stimulation effects both on CFT and regret. Furthermore, selfcritical rumination tendency also plays a crucial role when investigating the effects of tDCS intervention on psychophysiological measurements. In fact, prefrontal anodal left stimulation was found to attenuate skin conductance reactivity to the various choice outcomes exclusively among individuals prone to self-critical rumination. In other words, the Authors speculated that, in rumination-inclined individuals, anodal tDCS could decrease emotional arousal as indicated by CFT attenuation and by reduced pyshiological responsiveness. Moreover, anodal left tDCS was found to modulate differential reactivity of heart rate in rumination-prone individuals.

At biological level, this is in line with other tDCS studies in which neural correlates of ruminative thoughts improvements induced by tDCS seemed to be exerted on the modulation of left DLPFC activity, i.e., an increased left prefrontal cortical perfusion after MRI scan ${ }^{[17]}$. Intriguingly, tDCS effects on ruminations becomes evident even only through the application of a single tDCS session, unlike what is expected to detect changes in mood [17].

Recently, the growing interest of tDCS application on rumination was debated in a systematic review, including 9 original sham-controlled studies ${ }^{[18]}$. Of notice, only two studies comprised clinical samples, i.e. GeneralizedAnxiety Disorder ${ }^{[19]}$ and Major Depressive Disorder ${ }^{[20]}$, while the others relied on healthy volunteers. Summing up, it was showed that five out of the nine studies reported significant alleviation of rumination by tDCS: particularly, anodal stimulation of left DLPFC significantly reduced state rumination, as compared to sham stimulation [17] [21], while bihemispheric tDCS-montage (anodal right coupled with cathodal left DLPFC) was found to increase state rumination, as compared to the opposite tDCS-montage 
and sham stimulation [22]. Regarding electrodes placement, it was found that anodal stimulation was mostly applied over the left DLPFC (eight out of nine studies), confirming previous observations about prefrontal cortex involvement in rumination process. As stated by Hoebeke et al. (2021) [18], beyond protocols and stimulation parameters heterogeneity, the main limitation of the current literature is the lack of studies assessing the efficacy and the longitudinal effects of repeated tDCS sessions in clinical ruminative-prone populations.

Nevertheless, since ruminations could be described as a major cognitive-affective thought process that, with a transdiagnostic value, predispose to development and maintenance of psychiatric disorders, it could be argued that rumination represents an important emerging line of research for neurostimulation interventions, demonstrating how these techniques may act by modulating emotion and affective states influence exerted on different cognitive processes. In this context, an intriguing example is the modulation of the Cognitive Control Network (CCN), a regulatory system that controls the operation of cognitive and emotional systems to enable the individual to accomplish goals ${ }^{[23]}$. Since cognitive control impairments are widely observed in psychiatric disorders, contributing deficient daily functioning [24], and given that the DLPFC is a key node of the CCN, one could argue that neuromodulation interventions on this brain region could represent an interesting approach for treatment of psychiatric disorders, thus enhancing cognitive control and contributing to efficacious emotion regulation strategies (such as reappraisal, suppression, and rumination) by the modulation of aversive or irrelevant affective material [25] [26] [27] [28]. Among psychiatric disorders, in addition to cognitive control improvements observed in ruminant depressed patients [29], an emerging interest concerns tDCS application in Borderline Personality Disorder (BPD). Given the notion that emotion regulation disturbance is the primarily core feature of BPD [30], rumination, in turn, represents one of the principal dysfunctional affective strategies [31][32]. Hence, filling the gap between emotion and behavioral dysregulation, the Emotional Cascade Theory posits that aversive emotional states induce ruminations, which in turn increase the intensity of emotional distress through vicious ruminative cycles, until dysregulated behaviors are engaged to down-regulating emotional distress, distracting attention from aversive affects ${ }^{[33]}$. Thus, negative outcomes of rumination are associated with clinical manifestations of BPD, such as compensatory impulsive behaviors, including non-suicidal self-injury and aggressive conducts, binging on food, substance abuse ${ }^{[34]}$. Particularly, anger and depressive ruminations were found to be significantly associated to aggression and self-harming proneness, respectively ${ }^{[35]}$.

In this context, a rising number of tDCS studies in BPD, albeit characterized by heterogeneous protocol designs, have demonstrated innovative evidence of efficacy in the modulation of impulsive-aggressive behaviors and anger [36], as well as in executive dysfunctions, emotional dysregulation, and emotional processing [37], leading the Authors to conclude that DLPFC stimulation may act by enhancing prefrontal activity through cognitive control empowerment and down-regulating subcortical hyperactivity. Moreover, it should be noted how these substantial results were obtained through the applications of repeated tDCS sessions, while other studies on the cognitive control of affective states and consisting of a single daily 
session, did not clearly confirm these results [38] [39].

In conclusion, although tDCS literature in the field of ruminations has involved so far a limited number of clinical populations, we believe that it would be worthwhile to plan targeted interventions in BPD patients to improve the dysfunctional process of anger and depressive ruminations, with the aim of boost cognitive control over dysregulated emotions and, therefore, over related dysfunctional behavior.

\section{References}

1. `Martin, L. L., \& Tesser, A.. (1996). Some ruminative thoughts. In R. S. Wyer Jr. (Ed.). Hillsdale, NJ: Erlbaum.

2. `Edward R. Watkins. (2008). Constructive and unconstructive repetitive thought.. Psychological Bulletin, vol. 134 (2), 163-206. doi:10.1037/0033-2909.134.2.163.

3. a, bMaria Luca. (2019). Maladaptive Rumination as a Transdiagnostic Mediator of Vulnerability and Outcome in Psychopathology. JCM, vol. 8 (3), 314. doi:10.3390/jcm8030314.

4. `Susan Nolen-Hoeksema, Eric Stice, Emily Wade, Cara Bohon. (2007). Reciprocal relations between rumination and bulimic, substance abuse, and depressive symptoms in female adolescents.. Journal of Abnormal Psychology, vol. 116 (1), 198-207. doi:10.1037/0021-843x.116.1.198.

5. `Thomas Ehring, Edward R. Watkins. (2008). Repetitive Negative Thinking as a Transdiagnostic Process. International Journal of Cognitive Therapy, vol. 1 (3), 192-205. doi:10.1521/ijct.2008.1.3.192.

6. `Edward A. Selby, Michael D. Anestis, Thomas E. Joiner. (2008). Understanding the relationship between emotional and behavioral dysregulation: Emotional cascades. Behaviour Research and Therapy, vol. 46 (5), 593-611. doi:10.1016/j.brat.2008.02.002.

7. `Edward R. Watkins, Henrietta Roberts. (2020). Reflecting on rumination: Consequences, causes, mechanisms and treatment of rumination. Behaviour Research and Therapy, vol. $127,103573$. doi:10.1016/j.brat.2020.103573.

8. Evi De Lissnyder, Ernst H.W. Koster, Liesbet Goubert, Thomas Onraedt, et al. (2012). Cognitive control moderates the association between stress and rumination. Journal of Behavior Therapy and Experimental Psychiatry, vol. 43 (1), 519-525. doi:10.1016/j.jbtep.2011.07.004.

9. ^Rudi De Raedt, Paula T. Hertel, Edward R. Watkins. (2015). Mechanisms of Repetitive Thinking. Clinical Psychological Science, vol. 3 (4), 568-573. doi:10.1177/2167702615584309.

10. 'Yingkai Yang, Songfeng Cao, Grant S. Shields, Zhaojun Teng, et al. (2016). The relationships between rumination and core executive functions: A meta-analysis. Depress Anxiety, vol. 34 (1), 37-50. doi:10.1002/da.22539.

11. `Jutta Joormann, K. Lira Yoon, Ulrike Zetsche. (2007). Cognitive inhibition in depression. Applied and Preventive Psychology, vol. 12 (3), 128-139. doi:10.1016/j.appsy.2007.09.002.

12. ^Forster, S., Nunez Elizalde, A.O., Castle, E., Bishop, S.J.,. (2015). Unraveling the Anxious Mind: Anxiety, Worry, and Frontal Engagement in Sustained Attention Versus Off-Task Processing. Cereb. Cortex, vol. 25, 609-618. 
13. ${ }^{a}$, bai Epstude, Neal J. Roese. (2008). The Functional Theory of Counterfactual Thinking. Pers Soc Psychol Rev, vol. 12 (2), 168-192. doi:10.1177/1088868308316091.

14. `Min-Fang Kuo, Michael A. Nitsche. (2012). Effects of Transcranial Electrical Stimulation on Cognition. Clin EEG Neurosci, vol. 43 (3), 192-199. doi:10.1177/1550059412444975.

15. M Marine Mondino, Djamila Bennabi, Emmanuel Poulet, Filipe Galvao, et al. (2014). Can transcranial direct current stimulation (tDCS) alleviate symptoms and improve cognition in psychiatric disorders?. The World Journal of Biological Psychiatry, vol. 15 (4), 261-275. doi:10.3109/15622975.2013.876514.

16. `Jens Allaert, Rudi De Raedt, Frederik M. van der Veen, Chris Baeken, et al. (2021). Prefrontal tDCS attenuates counterfactual thinking in female individuals prone to self-critical rumination. Sci Rep, vol. 11 (1). doi:10.1038/s41598-021-90677-7.

17. a, b, cChris Baeken, Jonathan Remue, Marie-Anne Vanderhasselt, Andre R. Brunoni, et al. (2017). Increased left prefrontal brain perfusion after MRI compatible tDCS attenuates momentary ruminative self-referential thoughts. Brain Stimulation, vol. 10 (6), 1088-1095. doi:10.1016/j.brs.2017.09.005.

18. ${ }^{a}$, bYorgo Hoebeke, Olivier Desmedt, Betül Özçimen, Alexandre Heeren. (2021). The impact of transcranial Direct Current stimulation on rumination: A systematic review of the sham-controlled studies in healthy and clinical samples. Comprehensive Psychiatry, vol. $106,152226$. doi:10.1016/j.comppsych.2021.152226.

19. `Fariba Sadeghi Movahed, Jaber Alizadeh Goradel, Asghar Pouresmali, Mehri Mowlaie. (2018). Effectiveness of Transcranial Direct Current Stimulation on Worry, Anxiety, and Depression in Generalized Anxiety Disorder: A Randomized, Single-Blind Pharmacotherapy and Sham-Controlled Clinical Trial. Iran J Psychiatry Behav Sci, vol. 12 (2). doi:10.5812/ijpbs.11071.

20. `Marie-Anne Vanderhasselt, Rudi De Raedt, Victoria Namur, Paulo A. Lotufo, et al. (2015). Transcranial electric stimulation and neurocognitive training in clinically depressed patients: A pilot study of the effects on rumination. Progress in Neuro-Psychopharmacology and Biological Psychiatry, vol. 57 , 93-99. doi:10.1016/j.pnpbp.2014.09.015.

21. ^Rudi De Raedt, Jonathan Remue, Tom Loeys, Jill M. Hooley, et al. (2017). The effect of transcranial direct current stimulation of the prefrontal cortex on implicit self-esteem is mediated by rumination after criticism. Behaviour Research and Therapy, vol. 99 , 138-146. doi:10.1016/j.brat.2017.10.009.

22. 'Kelley, N.J., Hortensius, R., Harmon-Jones, E. (2013). When Anger Leads to Rumination: Induction of Relative Right Frontal Cortical Activity With Transcranial Direct Current Stimulation Increases AngerRelated Rumination.. Psychol. Sci., vol. 24, 475-481 .

23. `Tara A. Niendam, Angela R. Laird, Kimberly L. Ray, Y. Monica Dean, et al. (2012). Meta-analytic evidence for a superordinate cognitive control network subserving diverse executive functions. Cogn Affect Behav Neurosci, vol. 12 (2), 241-268. doi:10.3758/s13415-011-0083-5.

24. `Mark J. Millan, Yves Agid, Martin Brüne, Edward T. Bullmore, et al. (2012). Cognitive dysfunction in psychiatric disorders: characteristics, causes and the quest for improved therapy. Nat Rev Drug Discov, vol. 11 (2), 141-168. doi:10.1038/nrd3628. 
25. `Melanie Feeser, Kristin Prehn, Philipp Kazzer, Aditya Mungee, et al. (2014). Transcranial Direct Current Stimulation Enhances Cognitive Control During Emotion Regulation. Brain Stimulation, vol. 7 (1), 105112. doi:10.1016/j.brs.2013.08.006.

26. 'Christian Plewnia, Philipp A Schroeder, Larissa Wolkenstein. (2015). Targeting the biased brain: noninvasive brain stimulation to ameliorate cognitive control. The Lancet Psychiatry, vol. 2 (4), 351-356. doi:10.1016/s2215-0366(15)00056-5.

27. `Crystal Lantrip, Faith M. Gunning, Laura Flashman, Robert M. Roth, et al. (2017). Effects of Transcranial Magnetic Stimulation on the Cognitive Control of Emotion. J ECT, vol. 33 (2), 73-80. doi:10.1097/yct.0000000000000386.

28. A Ariane Wiegand, Anja Sommer, Vanessa Nieratschker, Christian Plewnia. (2019). Improvement of cognitive control and stabilization of affect by prefrontal transcranial direct current stimulation (tDCS). Sci Rep, vol. 9 (1). doi:10.1038/s41598-019-43234-2.

29. ' Larissa Wolkenstein, Christian Plewnia. (2013). Amelioration of Cognitive Control in Depression by Transcranial Direct Current Stimulation. Biological Psychiatry, vol. 73 (7), 646-651. doi:10.1016/j.biopsych.2012.10.010.

30. 'Linehan, M. M. (1993). Diagnosis and treatment of mental disorders. Cognitive-behavioral treatment of borderline personality disorder. Guilford Press.

31. ^Ruth A. Baer, Jessica R. Peters, Tory A. Eisenlohr-Moul, Paul J. Geiger, et al. (2012). Emotion-related cognitive processes in borderline personality disorder: A review of the empirical literature. Clinical Psychology Review, vol. 32 (5), 359-369. doi:10.1016/j.cpr.2012.03.002.

32. ^Liliana Dell'Osso, Ivan M Cremone, Barbara Carpita, Valerio Dell'Oste, et al. (2019). $\leq p>$ Rumination, posttraumatic stress disorder, and mood symptoms in borderline personality disorder $\langle/ p\rangle$. NDT, vol. Volume 15, 1231-1238. doi:10.2147/ndt.s198616.

33. `Edward A. Selby, Michael D. Anestis, Theodore W. Bender, Thomas E. Joiner. (2009). An exploration of the emotional cascade model in borderline personality disorder.. Journal of Abnormal Psychology, vol. 118 (2), 375-387. doi:10.1037/a0015711.

34. ^Rebecca Meaney, Penelope Hasking, Andrea Reupert. (2016). Borderline Personality Disorder Symptoms in College Students: The Complex Interplay between Alexithymia, Emotional Dysregulation and Rumination. PLoS ONE, vol. 11 (6), e0157294. doi:10.1371/journal.pone.0157294.

35. ^Francesca Martino, Gabriele Caselli, Jessica Di Tommaso, Sandra Sassaroli, et al. (2017). Anger and depressive ruminations as predictors of dysregulated behaviours in borderline personality disorder. Clin Psychol Psychother, vol. 25 (2), 188-194. doi:10.1002/cpp.2152.

36. `Jacopo Lisoni, Paola Miotto, Stefano Barlati, Stefano Calza, et al. (2020). Change in core symptoms of borderline personality disorder by tDCS: A pilot study. Psychiatry Research, vol. $291,113261$. doi:10.1016/j.psychres.2020.113261.

37. ^Parviz Molavi, Samaneh Aziziaram, Sajjad Basharpoor, Akbar Atadokht, et al. (2020). Repeated transcranial direct current stimulation of dorsolateral-prefrontal cortex improves executive functions, 
cognitive reappraisal emotion regulation, and control over emotional processing in borderline personality disorder: A randomized, sham-controlled, parallel-group study. Journal of Affective Disorders, vol. 274 , 93-102. doi:10.1016/j.jad.2020.05.007.

38. `Lars Schulze, Maren Grove, Sascha Tamm, Babette Renneberg, et al. (2019). Effects of transcranial direct current stimulation on the cognitive control of negative stimuli in borderline personality disorder. Sci Rep, vol. 9 (1). doi:10.1038/s41598-018-37315-x.

39. `Larissa Wolkenstein, Felicitas Rombold-Bruehl, Tiffany Bingmann, Anja Sommer, et al. (2021).

Challenging control over emotions in borderline personality disorder - a tDCS study. Neuropsychologia, vol. 156 , 107850. doi:10.1016/j.neuropsychologia.2021.107850. 